\title{
Development of a neutralization assay based on the pseudotyped chikungunya virus of a Korean isolate
}

\author{
Woo-Chang Chung ${ }^{1}$, Kwang Yeon Hwang ${ }^{2}$, \\ Suk-Jo Kang , Jae-Ouk Kim ${ }^{4}$, \\ and Moon Jung Song ${ }^{1 \star}$ \\ ${ }^{1}$ Virus-Host Interactions Laboratory, Department of Biosystems and \\ Biotechnology, Division of Biotechnology, College of Life Sciences and \\ Biotechnology, Korea University, Seoul 02841, Republic of Korea \\ ${ }^{2}$ Structural Proteomics Laboratory, Department of Biosystems and \\ Biotechnology, Division of Biotechnology, College of Life Sciences and \\ Biotechnology, Korea University, Seoul 02841, Republic of Korea \\ ${ }^{3}$ Department of Biological Sciences, Korea Advanced Institute of Science \\ and Technology, Daejeon 34141, Republic of Korea \\ ${ }^{4}$ Molecular Immunology, Science, International Vaccine Institute, \\ Seoul 08826, Republic of Korea \\ (Received Aug 7, 2019 / Revised Oct 7, 2019 / Accepted Oct 7, 2019)
}

The Chikungunya virus (CHIKV) belongs to the Alphavirus genus of Togaviridae family and contains a positive-sense single stranded RNA genome. Infection by this virus mainly causes sudden high fever, rashes, headache, and severe joint pain that can last for several months or years. CHIKV, a mosquito-borne arbovirus, is considered a re-emerging pathogen that has become one of the most pressing global health concerns due to a rapid increase in epidemics. Because handling of CHIKV is restricted to Biosafety Level 3 (BSL-3) facilities, the evaluation of prophylactic vaccines or antivirals has been substantially hampered. In this study, we first identified the whole structural polyprotein sequence of a CHIKV strain isolated in South Korea (KNIH/2009/77). Phylogenetic analysis showed that this sequence clustered within the East/ Central/South African CHIKV genotype. Using this sequence information, we constructed a CHIKV-pseudotyped lentivirus expressing the structural polyprotein of the Korean CHIKV isolate (CHIKVpseudo) and dual reporter genes of green fluorescence protein and luciferase. We then developed a pseudovirus-based neutralization assay (PBNA) using CHIKVpseudo. Results from this assay compared to those from the conventional plaque reduction neutralization test showed that our PBNA was a reliable and rapid method to evaluate the efficacy of neutralizing antibodies. More importantly, the neutralizing activities of human sera from CHIKVinfected individuals were quantitated by PBNA using CHIKVpseudo. Taken together, these results suggest that our PBNA for CHIKV may serve as a useful and safe method for testing the neutralizing activity of antibodies against CHIKV in BSL-2 facilities.

${ }^{\star}$ For correspondence. E-mail: moonsong@korea.ac.kr; Tel.: +82-2-32903019; Fax: +82-2-3290-3040

Copyright (c) 2020, The Microbiological Society of Korea
Keywords: Chikungunya virus, Korean isolate, Pseudovirus, Neutralization assay, Human serum

\section{Introduction}

Chikungunya virus (CHIKV) belongs to the Alphavirus genus of the Togaviridae family and is an arbovirus that can be transmitted by mosquitoes such as Aedes aegypti and Aedes albopictus. Its infection causes chikungunya fever, characterized by a high fever, rashes, headaches, and severe joint pain that can last for several months or years. CHIKV is considered a re-emerging human pathogen and has caused millions of cases of infection around the world (Schwartz and Albert, 2010; Thiboutot et al., 2010). As a tropical disease, CHIKV epidemic has mostly been reported in regions near and including the Indian Ocean, Pacific Islands, and the Americas (Morrison, 2014). However, global warming and international transportation have increased the risk of spreading CHIKV infections to non-endemic regions, including South Korea, where travel-associated or imported cases of CHIKV infection have been reported (Cha et al., 2013; Hwang and Lee, 2015; Yeom, 2017). In addition, there may be a potential risk of local transmission of CHIKV, because the habitat of Aedes albopictus has been increased significantly in South Korea (Chang el al., 2018).

The complete genome of CHIKV is a linear, positive-sense, single-stranded RNA with 11,805 nucleotides that encodes two polyproteins (Schwartz and Albert, 2010; Morrison, 2014). One polyprotein consists of the nonstructural proteins nsP1, $\mathrm{ns} 2$, nsP3, and nsP4 that are responsible for viral genome replication and viral gene transcription in infected cells. The other polyprotein consists of the capsid, E3, E2, 6K, and E1 structural proteins that are responsible for virion formation. The translated structural polyprotein is autocatalytically processed into capsid protein $\mathrm{C}, \mathrm{E} 1,6 \mathrm{~K}$, and p62. The capsid protein $\mathrm{C}$ encapsidates viral genomic RNA, while the glycoproteins E1 and p62 interact to form heterodimers that subsequently trimerize into a viral spike in the endoplasmic reticulum (Jose et al., 2009; Li et al., 2010). The glycoprotein p62 is then cleaved into E2 and E3 by cellular furin. Glycoprotein E2 is important for receptor binding during viral entry, while glycoprotein E1 is responsible for membrane fusion during viral infection (Bréhin et al., 2008; Kuo et al., 2012). Although CHIKV glycoprotein E3 is released after budding and not associated with the mature virion, glycoprotein E3 facilitates E1-p62 heterodimerization and prevents the exposure of the E1 fusion loops from premature fusogenic activity (Mulvey and Brown, 1995; Carleton et al., 1997; Solignat et al., 2009; Voss et al., 2010; Snyder and Muk- 
hopadhyay, 2012; Yap et al., 2017). In addition, the E2 protein has been shown to be a major epitope for neutralizing antibodies (Voss et al., 2010).

Despite the increasing global concerns about the worldwide reemergence of CHIKV, there is no approved antiviral treatment for CHIKV infection or vaccine against CHIKV (Jain et al., 2008; Tharmarajah et al., 2017). Multiple technology platforms have been applied to develop CHIKV vaccines using live attenuated viruses, inactivated viruses, recombinant viruses, chimeric alphaviruses, or virus-like particles (VLP), although such vaccines are still in various stages of the clinical or pre-clinical processes (Weaver et al., 2012; Powers, 2018). In addition, vaccine efficacy is often evaluated using the conventional plaque reduction neutralization test (PRNT) (Azami et al., 2016). PRNT determines the neutralizing antibody titer of a test sample by counting the number of plaques formed by cytopathic effects of viral infection. However, it is difficult to handle live, biohazardous CHIKV because its use requires a biosafety level-3 (BSL-3) facility, therefore an alternative assay to evaluate vaccine efficacy may facilitate vaccine development.

In this study, we aimed to develop a pseudovirus-based neutralization assay (PBNA) using KNIH/2009/77, a Korean isolate of CHIKV (Cha et al., 2013). A CHIKV-pseudotyped virus (CHIKVpseudo) expressing the structural polyprotein of CHIKV, was constructed using a lentiviral vector, which expressed both luciferase and green fluorescence protein (GFP) reporters. Validation and comparisons of PBNA results with those from conventional PRNT indicate that the PBNA using CHIKVpseudo may be a safe, rapid, and reliable neutralization assay for evaluating the neutralizing activity of anti-sera against CHIKV.

\section{Materials and Methods}

\section{Cells, viruses, antibodies, and human sera}

Vero, HeLa, BHK-21, and HEK293T cells were cultured in complete Dulbecco's modified Eagle's medium (HyClone) containing $10 \%$ fetal bovine serum (FBS; HyClone) and supplemented with $100 \mathrm{U} / \mathrm{ml}$ penicillin and $100 \mu \mathrm{g} / \mathrm{ml}$ streptomycin (HyClone). KNIH/2009/77, the Korean isolated strain of CHIKV, was provided by the National Culture Collection for Pathogens, Korea. A neutralizing antibody against CHIKV E2 protein (anti-E2; chk-265 from Absolute Antibody Ltd.) was used for plaque reduction neutralization tests (PRNTs) and pseudovirus-based neutralization assays (PBNAs) (Pal et al., 2013), while a neutralizing antibody against the spike protein of the Middle East Respiratory Syndrome coronavirus (MERS-CoV) (Sino Biological Inc., Cat: 40069-R273) was used as a control for PBNA specificity. Human sera were obtained from a study titled "Burden of dengue infection in children and adults of Ouagadougon, Burkina Faso" with informed consent of the subjects. The subjects authorized researchers for the utilization of the left-over blood samples to identify other pathogens to study disease transmission in this area. This additional study was approved by the Institutional Review Board of International Vaccine Institute (IVI IRB protocol number: 2014-008).

\section{Sequencing and phylogenetic analysis of structural proteins}

KNIH/2009/77 infected BHK-21 cells were harvested with TRI reagent (MRC). RNA was extracted using a chloroform extraction method. The cDNAs were synthesized using the RevertAid first-strand cDNA synthesis kit (Invitrogen) with oligo dT primers. The synthesized cDNA was used as template DNA for PCR amplification of the structural polyprotein coding region, which was then cloned into the pCMV2FLAG vector (pCMV2-FLAG-CHIKVst) using the following primers: F: $5^{\prime}$-CTTGCGGCCGCGATGGAGTTCATC CCAACCCAAACT-3' and R: 5' -TATATTGGATCCTTAG TGCCTGCTGAACGACACGC-3'. Underlined letters indicate the Not I and BamHI restriction enzyme sites used for cloning. To sequence the cloned structural polyprotein, additional primers targeting the Capsid gene $\left(5^{\prime}\right.$-GCCATCCC AGTTATGTGCCTGTTGG-3') and the E2 gene ( $5^{\prime}$-CAG TATAACTCCCCCTCTGGTCACGCGT-3') were used and the sequence submission can be found in GenBank. The nucleotide sequences of structural polyprotein coding genes from $16 \mathrm{CHIKV}$ isolates were selected for phylogenetic analysis (Table 1). Amino acid sequences were aligned by the Clustal Omega multiple sequence alignment program. The neighbor-joining algorithm and the maximum composite likelihood model were used for tree construction by MEGA 7 software (Tamura et al., 2011). The reliability of the analysis was evaluated by a bootstrap test with 2,000 replicates.

\section{Production of pseudotyped CHIKV}

The expression of structural proteins of CHIKV from pCMV2FLAG-CHIKVst was tested. The plasmid was transfected into HEK293T cells using polyethyleneimine (Fukumoto et al., 2010). At $24 \mathrm{~h}$ post-transfection, cells were harvested and analyzed by western blot as described below. To produce CHIKVpseudo, HEK293T cells were transfected with pCMV2FLAG-CHIKVst, psPAX2 (a gift from Dr. Seungmin Hwang, The University of Chicago, IL, USA), and Luc2P pLVX-IRESZsGreen1, which is able to express two reporter genes, luciferase and GFP (a gift from Dr. Nam-Hyuk Cho, Seoul Na-

\begin{tabular}{lcc}
\multicolumn{1}{c}{ Table 1. CHIKV strains used in phylogenetic analysis } \\
\hline \multicolumn{1}{c}{ Strain } & Geographic origin & Sampling year \\
\hline ROSS & Tanzania & 1953 \\
PM2951 & Senegal & 1966 \\
18211 & South Africa & 1976 \\
HD 180760 & Senegal & 2005 \\
La Reunion & La Reunion & 2006 \\
MY/06/37348 & Malaysia & 2006 \\
SL-CK1 & Sri Lanka & 2007 \\
0706aTw & Taiwan & 2007 \\
SGEHICHD 122508 & Singapore & 2008 \\
MY/08/065 & Malaysia & 2008 \\
Mal2008 & Malaysia & 2008 \\
TN06410 & India & 2010 \\
VE53 20 & Trinidad and Tobago & 2014 \\
IND-73-MH5 & India & Unknown \\
IND-63-WB1 & India & Unknown \\
AF15561 & Thailand & Unknown \\
KNIH/2009/77 & Korea & 2009 \\
\hline
\end{tabular}


tional University, South Korea). pMD2.G encoding VSV-G (a gift from Dr. Seungmin Hwang, The University of Chicago, IL, USA) was used for control pseudotyped virus production. Cell media was changed around $16 \mathrm{~h}$ post-transfection. The supernatant containing cultured viruses was harvested at 3 days after the media change, then filtered using a $0.2 \mu \mathrm{m}$ syringe filter. The virus stock was aliquoted in small volumes and stored at $-80^{\circ} \mathrm{C}$ until use. For titration of the pseudotyped CHIKV, HeLa cells were infected with CHIKVpseudo by incubation with virus-containing media for 2 days. The number of GFP expressing cells were counted using FACS (FACSCalibur, BD Biosciences) to calculate the titer of infectious CHIKVpseudo. Cells were then harvested and analyzed by western blotting to evaluate structural protein expression. SDS-PAGE resolved lysates were transferred to a polyvinylidene fluoride (PVDF) membrane and probed with primary antibodies against FLAG-M2 (1:2,000; Sigma), E2 (1:500; Absolute Antibody Ltd.), or a-tubulin (1:2,000; Sigma). Antimouse immunoglobulin $\mathrm{G}$ antibody conjugated with horseradish peroxide (1:5000, Santa Cruz Biotechnology) was used as a secondary antibody. Detection was performed using ECL plus western blotting detection reagents (ELPIS) and an LAS4000 chemiluminescent image analyzer (Fujifilm).

\section{Plaque reduction neutralization test (PRNT)}

The ability of an antibody to neutralize CHIKV was determined using the PRNT with Vero cells. We used a neutralizing antibody against CHIKV E2 protein (anti-E2; chk-265 from Absolute Antibody Ltd.) as a reference antibody (Pal et al., 2013) and experiments were done in triplicate. Threefold serial dilutions of stock antibody were made with DMEM containing 5\% heat-inactivated FBS-containing, then mixed with a fixed amount of CHIKV (KNIH/2009/77, $100 \mathrm{PFU} /$ well in 12 well plates) and incubated at $37^{\circ} \mathrm{C}$ for $1 \mathrm{~h}$. After incubation, a Vero cell monolayer was infected with the virus/ antibody mixtures and incubated at $37^{\circ} \mathrm{C}$ for $90 \mathrm{~min}$ to adsorb the virus. Next, after removing the inoculum, the overlay media containing $1 \%$ methylcellulose was added to the infected Vero cells. After 4 days of incubation, cells were fixed and stained with $2 \%$ crystal violet in $20 \%$ ethanol. Plaques were counted to calculate the inhibitory concentration of the antibody against CHIKV. The neutralization curve and the effective concentration of $50 \%$ neutralization $\left(\operatorname{LogIC} \mathrm{C}_{50}\right)$ were calculated using GraphPad Prism (GraphPad).

\section{Pseudovirus-Based Neutralization Assay (PBNA) using CHIKV- pseudo}

PBNA experiments were done in triplicate. For the PBNA, three-fold serial dilutions of the anti-E2 antibody were mixed with CHIKVpseudo solution at an estimated titer of 100 GFP unit/well and then incubated at $4^{\circ} \mathrm{C}$ for $1 \mathrm{~h}$. All antibody dilution was performed with DMEM containing 5\% heat-inactivated FBS. Then, a HeLa cell monolayer was infected with the CHIKVpseudo/antibody mixtures and incubated at $37^{\circ} \mathrm{C}$ for $4 \mathrm{~h}$. Infected cells were cultured in complete media for additional 2 days. Cells were then harvested and analyzed by a luciferase activity using the luciferase reporter assay system (Promega) following manufacturer's instructions. The neutralization curve and the effective concentration for $50 \%$ neutralization $\left(\operatorname{LogIC} \mathrm{C}_{50}\right)$ were calculated using GraphPad Prism.

\section{Results}

\section{Phylogenetic analysis of KNIH/2009/77 based on the whole structural polyprotein sequence}

Although not frequent, travel-associated cases of CHIKV infection have recently been increased and detected in 5\% of patients with dengue-like symptoms (Cha et al., 2013; Hwang and Lee, 2015; Yeom, 2017). KNIH/2009/77 was isolated from such a patient and deposited to the National Culture Collection for Pathogens (Cha et al., 2013). To further perform a genetic analysis of $\mathrm{KNIH} / 2009 / 77$, we amplified the structural polyprotein coding region from cDNA of $\mathrm{KNIH} / 2009 / 77$ using a reverse transcription system. The amplified structural polyprotein DNA of $3.7 \mathrm{~kb}$ in length

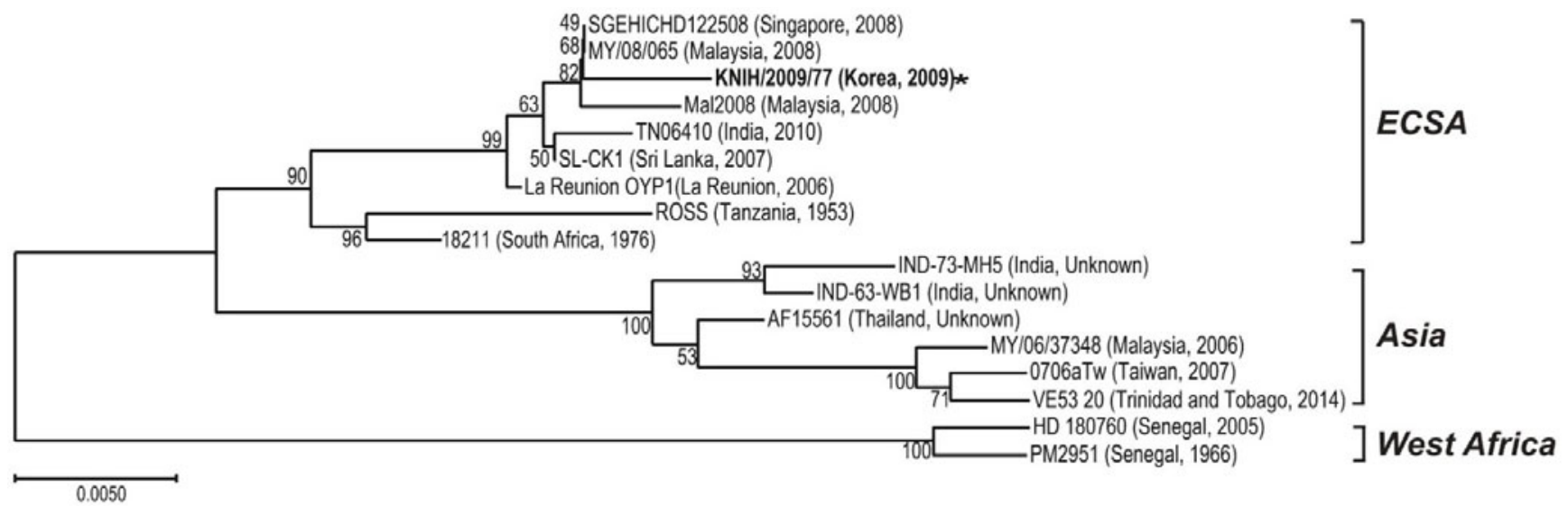

Fig. 1. Phylogenetic analysis of CHIKV strains based on the structure protein sequences. Phylogenetic relationships were inferred using the NeighborJoining method with whole structural polyprotein of CHIKV. The percentage of replicate trees in which the associated taxa clustered together in the bootstrap test (2,000 replicates) are shown next to the branches. The evolutionary distances were computed using the Poisson correction method. The position of the Korean Isolate, KNIH/2009/77, is indicated by bold letters and an asterisk. The names of clustered genotype strains are indicated. ECSA: East/ Central/South African. 
was cloned into an expression vector system (pCMV2-FLAGCHIKVst) and primers used to specifically amplify portions of the capsid (C) and E2 genes. An NCBI-BLAST search showed that the sequence of the structural polyprotein has 99\% sequence similarity with other CHIKV isolates belonging to the East/Central/South African (ECSA) genotype. The structural polyprotein sequence of KNIH/2009/77 was then aligned with sequences from Asian, West African, or ECSA genotypes using Clustal $\mathrm{W}$ alignment and the phylogenetic position of the KNIH/2009/77 isolate identified using MEGA7 software. The KNIH/2009/77 isolate clustered with ECSA genotypes and with isolates from Singapore and Malaysia (Table 1 and Fig. 1).

\section{Development of pseudovirus-based neutralization assays using CHIKVpseudo}

We have established a reliable plaque reduction neutraliza- tion test (PRNT) using KNIH/2009/77. However, because of the potential limitations of handling CHIKV in laboratories owing to its biohazard risk level, we set out to develop an alternative pseudovirus-based neutralization assay (PBNA) that may replace the conventional PRNT. A pseudotyped virus of KNIH/2009/77 (CHIKVpseudo) was designed to express the full-length structural polyprotein rather than individual structural proteins to enhance its infectivity (Hu et al., 2014). A schematic diagram of the structural polyprotein expression plasmid of KNIH/2009/77 (pCMV2-FLAG-CHIKVst) is shown in Fig. 2A. To produce CHIKVpesudo, pCMV2FLAG-CHIKVst was transfected into HEK293T cells with a lentivirus-based dual reporter vector (Luc2P-pLVX-IRESZsGreen1) and a packaging vector of lentivirus (psPAX2) (Fig. 2B). FLAG-tagged capsid protein was detected in HEK293T cells infected with CHIKVpseudo using anti-FLAG antibody (Fig. 2C). The expression of glycoprotein E2 was

(A)

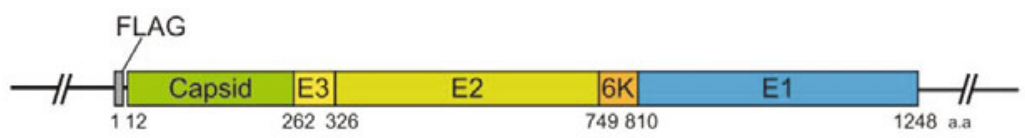

(B)

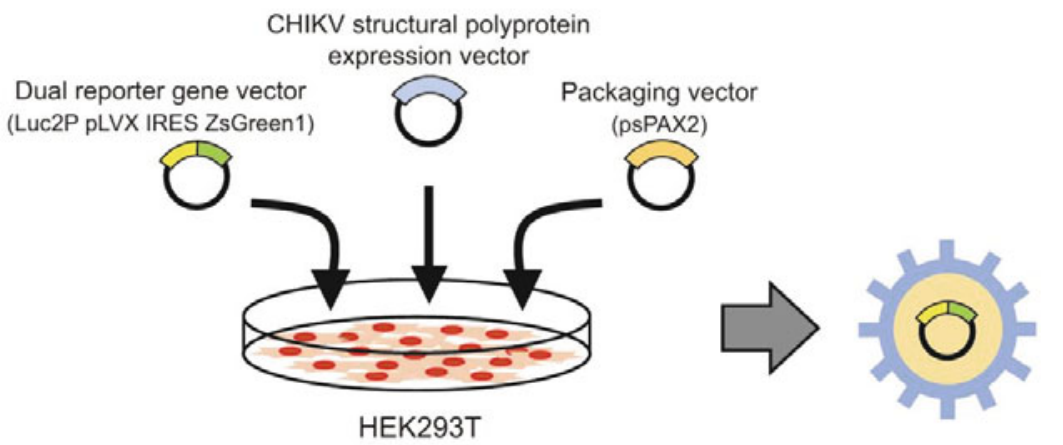

Transfect vector set

Harvest CHIKVpseudo

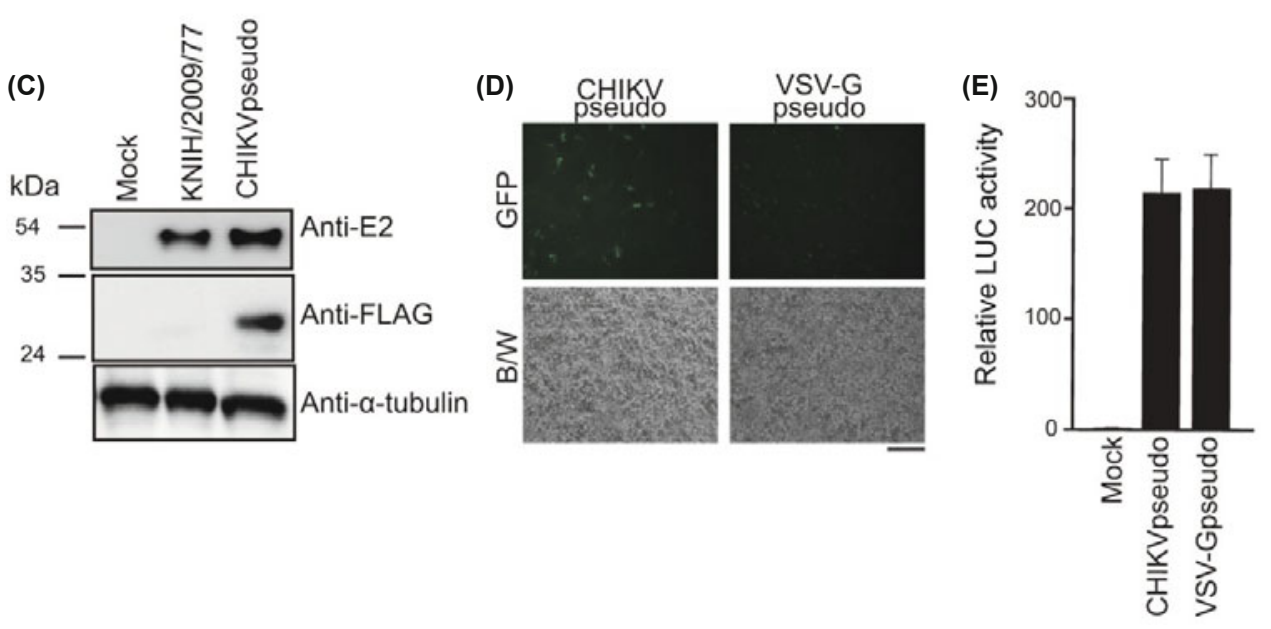

Fig. 2. Construction of a pseudotyped virus expressing the structural polyprotein of CHIKV (CHIKVpseudo). (A) A schematic diagram of the KNIH/2009/77 structural polyprotein from pCMV2-FLAG-CHIKVst. (B) The CHIKVpseudo construction scheme. (C) Expression of CHIKV proteins from the CHIKVpseudo-infected or KNIH/2009/77-infected cells. At $48 \mathrm{~h}$ post-infection by CHIKVpseudo (100 GFP unit/well) or KNIH/2009/77 (MOI=0.05), HEK293T cells were harvested and analyzed by western blot for CHIKV E2 and FLAG-Capsid expression. (D) GFP expression from CHIKVpseudo infected cells. HEK293T cells were infected with CHIKVpseudo then 2 days later GFP signal was examined. Lentivirus with VSV-G was used as control. Scale bar: $200 \mu \mathrm{m}$. (E) Luciferase activity from CHIKVpseudo infected cells. HeLa cells were infected with CHIKVpseudo then, 2 days later, cells were harvested for luciferase reporter assays. Lentivirus with VSV-G was used as control. 
(A)

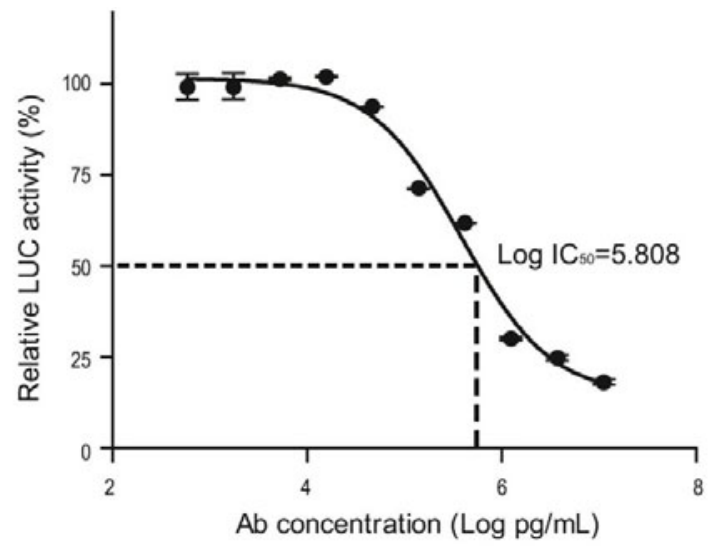

(B)

\begin{tabular}{c|c|c}
\hline & $\begin{array}{c}\text { PBNA with } \\
\text { CHIKVpseudo }\end{array}$ & $\begin{array}{c}\text { PRNT with } \\
\mathrm{KNIH} / 2009 / 77\end{array}$ \\
\hline $\begin{array}{c}\text { Calculated Log IC } \mathrm{S}_{0} \text { average } \\
(\mathrm{pg} / \mathrm{mL})\end{array}$ & $5.85 \pm 0.09$ & $5.68 \pm 0.06$ \\
\hline $\begin{array}{c}\text { Coefficient of variation (CV) } \\
(<25 \%)\end{array}$ & 1.09 & 0.98 \\
\hline
\end{tabular}

Fig. 3. Neutralization assays of anti-E2 antibody using CHIKVpseudo. (A) PBNA using CHIKVpseudo was performed with three-fold serial dilutions of anti-E2 antibody solution, starting from the concentration of $24 \mu \mathrm{g} / \mathrm{ml}$. Luciferase activity was measured at $48 \mathrm{~h}$ post-infection of CHIKVpseudo. In parallel, PRNT with KNIH/2009/77 was performed with three-fold serial dilutions of anti-E2 antibody solution. The plaque numbers were counted at 4 days post-infection. The effective concentration of $50 \%$ inhibition ( $\log$ $\mathrm{IC}_{50}$ ) of the PBNA or PRNT was calculated using GraphPad Prism. (A) The calculated Log $\mathrm{IC}_{50}$ of the PBNA for the anti-E2 antibody with CHIKVpseudo. (B) Validation report of the PNBA. The average of calculated $\log \mathrm{IC}_{50} \pm$ standard deviation is indicated. The PNBA of anti-E2 antibody solution using CHIKVpseudo was assessed for its precision, which is determined by the coefficient of variation (CV) and linearity, which is indicated by a regression coefficient $\left(R^{2}\right)$. Each value was compared with the validation report of the plaque reduction neutralization test (PRNT) with KNIH/2009/77. Calculated values were obtained from five independent experiments of PBNA using different batches of pseudovirus and three independent experiments of PRNT. also observed in CHIKVpseudo-transduced cells, as in KNIH/ 2009/77-infected cells using anti-E2 antibody. CHIKVpseudotransduced cells also showed expression of both the GFP and firefly luciferase dual reporter genes, allowing us to quantify the infectivity of CHIKVpseudo (Fig. 2D and E). Although both HEK293T cells and HeLa cells resulted in high efficiency in transduction of CHIKVpseudo, we set to use HeLa cells for PBNA, because HeLa cells were more durable for the process of PBNA, providing more reliable values (data not shown).

\section{Precision of PBNA with anti-E2 using CHIKVpseudo}

To check whether CHIKVpseudo can be utilized for a neutralization assay, we assessed the neutralizing activity of an antibody against the structural protein E2 (anti-E2), ranging the concentrations from $24 \mu \mathrm{g} / \mathrm{ml}$ to $1.2 \mathrm{ng} / \mathrm{ml}$, as produced from CHIKVpseudo infection. First, the concentration of antibody required to inhibit CHIKVpseudo infection by $50 \%\left(\mathrm{IC}_{50}\right)$ was calculated. CHIKVpseudo infection levels

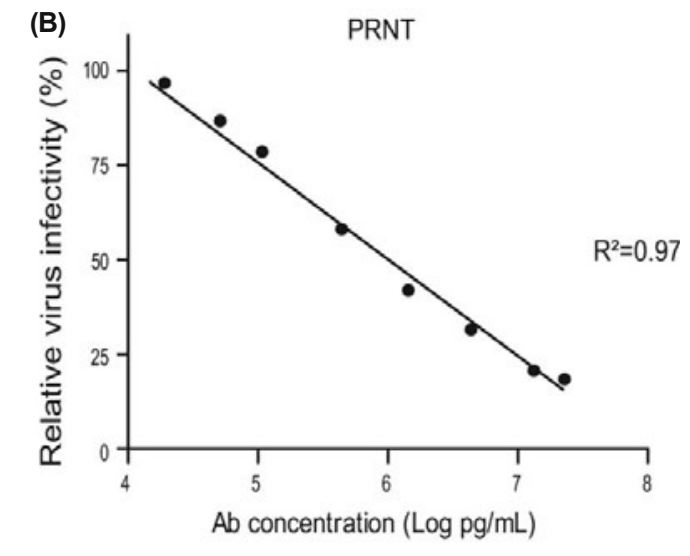

Average $\mathrm{R}^{2}=0.95 \pm 0.03$

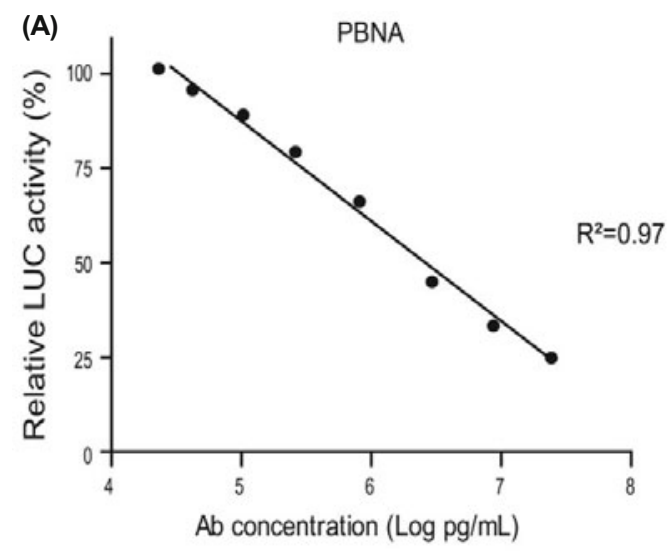

$\mathrm{R}^{2}=0.96 \pm 0.01$

Fig. 4. Linear regression analysis of the neutralization assay with CHIKVpseudo. PBNA using CHIKVpseudo was performed with three-fold serial dilutions of anti-E2 antibody solution, starting from the concentration of $24 \mu \mathrm{g} / \mathrm{ml}$. Luciferase activity was measured at $48 \mathrm{~h}$ post-infection of CHIKVpseudo. In parallel, PRNT with KNIH/2009/77 was performed with three-fold serial dilutions of anti-E2 antibody solution. The plaque numbers were counted at 4 days post-infection. The neutralization assay of anti-E2 antibody solution using CHIKVpseudo (A) and the PRNT (B) were assessed for linearity, indicated by the regression coefficient $\left(\mathrm{R}^{2}\right)$. The calculated values of average $\mathrm{R}^{2} \pm$ standard deviation were obtained from three independent experiments. 
were decreased by the anti-E2 in a dose-dependent manner and the $\log \mathrm{IC}_{50}$ of anti-E2 was estimated to be $5.808(\mathrm{pg} / \mathrm{ml})$ (Fig. 3A). We next compared the results of the neutralization assay using CHIKVpseudo with the conventional PRNT for $\mathrm{KNIH} / 2009 / 77$ to validate our results. The neutralizing activity of the same anti-E2 antibody was tested with PRNT for infection with $\mathrm{KNIH} / 2009 / 77$ and each calculated value was obtained from 3 independent experiments. PBNAs using anti-E2 and CHIKVpseudo elicited an average $\mathrm{LogIC}_{50}$ value of $5.85 \pm 0.09 \mathrm{pg} / \mathrm{ml}$ while a PRNT with KNIH/2009/77 yielded a $\operatorname{LogIC} \mathrm{C}_{50}$ of $5.68 \pm 0.06 \mathrm{pg} / \mathrm{ml}$. We then used the estimated values of $\operatorname{LogIC}_{50}$ from those 3 independent experiments to determine assay precision by calculating the coefficient of variation $(\mathrm{CV})$ for each assay. Neutralization assays with CHIKVpseudo showed a CV value of $1.09 \%$, which is similar to the PRNT CV of $0.98 \%$, indicating that PBNA is a sufficiently precise neutralization assay.

\section{Linearity of PBNA with anti-E2 using CHIKVpseudo}

When we used serial three-fold dilutions of the anti-E2 antibody, starting from the concentrations of $24 \mu \mathrm{g} / \mathrm{ml}$ in our PBNA, the linear regression analyses of the neutralizing activity showed linearity in the range of $5 \%$ to $80 \%$ infectivity reduction (Fig. 4). PBNAs with CHIKVpseudo yielded an average regression coefficient $\left(\mathrm{R}^{2}\right)$ value of 0.96 from 3 independent PBNAs (Fig. 4A). Similarly, conventional PRNTs yielded an average $R^{2}$ value of 0.95 (Fig. 4B). These results thus indicate that for both the neutralization assay with CHIKVpseudo and the PRNT, there is a significant correlation between the level of anti-E2 and the inhibition of CHIKVpseudo infectivity.

\section{Specificity of PBNA with anti-E2 using CHIKVpseudo}

To evaluate the specificity of PBNAs for anti-E2 targeting of $\mathrm{CHIKV}$, we compared the neutralizing ability of anti-E2 and an antibody against the spike protein of MERS-CoV (antispike), an unrelated protein. Serial three-fold dilutions of the antibody solution, starting from the concentrations of 24 $\mu \mathrm{g} / \mathrm{ml}$ were incubated with $100 \mathrm{GFP}$ unit of CHIKVpseudo per dilution and added to the cells for infection. Results from

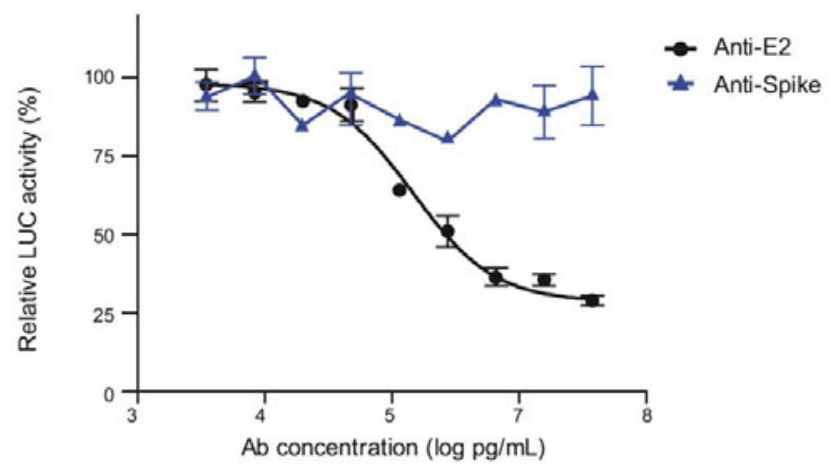

Fig. 5. Specificity of PBNA with CHIKVpseudo using antibody against E2 protein of CHIKV or spike protein of MERS. Three-fold serial dilutions of anti-E2 antibody or anti-spike protein of MERS-CoV antibody were mixed with the same volume of CHIKVpseudo solution and added to HeLa cells. Luciferase activity was measured at $48 \mathrm{~h}$ post-infection.

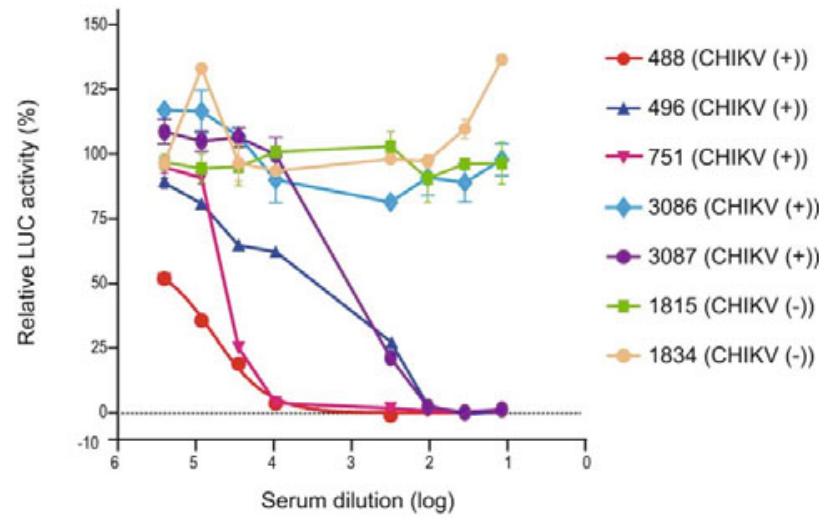

Fig. 6. Neutralization of CHIKVpseudo by human sera. PBNA using CHIKVpseudo was performed with three-fold serial dilutions of human sera. Luciferase activity was measured at $48 \mathrm{~h}$ post-infection of CHIKVpseudo. Five serum samples were obtained from CHIKV-positive patients (CHIKV $(+)$ serum No. 488, 496, 751, 3086 and 3087), while two serum samples were from CHIKV-negative patients (CHIKV(-) serum No. 1815 and 1834) as negative controls.

these infections indicated that the anti-E2 antibody inhibits CHIKVpseudo in a dose-dependent manner. However, the anti-spike antibody did not show dose-dependent inhibition (Fig. 5), indicating that CHIKVpseudo infection was specifically inhibited by the antibody against the CHIKV protein E2.

\section{Neutralization of CHIKVpseudo by CHIKV-infected human sera}

As previously reported, pseudotyped virus can be applied in detection of neutralizing antibodies in infected patients (Hu et al., 2014; Noranate et al., 2014). For this purpose, we tested the neutralization of CHIKVpseudo with serum samples from $5 \mathrm{CHIKV}$-positive individuals. These sera were only positive for CHIKV, but negative for all other mosquitoborne viruses including dengue virus (DENV), zika virus, yellow fever virus, West Nile virus, and Japanese encephalitis virus, as confirmed in ELISA (data not shown). Serum samples that were negative for all abovementioned mosquitoborne viruses were also used as negative controls (CHIKVnegative). Four out of five serum samples from CHIKVpositive individuals neutralized CHIKVpseudo in a dilutiondependent manner to various degrees, while none of serum samples from CHIKV-negative controls did (Fig. 6). This result indicates that CHIKVpseudo can be applied to measure the neutralizing activity of clinical samples.

\section{Discussion}

CHIKV, a member of the Alphavirus genus of the Togaviridae family, is a tropical disease-causing virus that is transmitted by mosquitoes. Global warming and frequent international travel have increased the risk of mosquito-borne diseases globally, and CHIKV is no exception. Over millions of CHIKV infection cases have been reported in more than 50 countries, suggesting that CHIKV has re-emerged as a global pathogen 
(Morrison, 2014). There are three strains of CHIKV based on their partial E1 sequences: East/Central/South African (ECSA), West African, and Asian (Powers et al., 2000; Rezza et al., 2007; Volk et al., 2010). Each strain has been associated with regional epidemics, but the ECSA strain caused an extensive nationwide outbreak in ectopic areas such as Malaysia in 2008 (Sam et al., 2012; Morrison, 2014). Recently in South Korea, the cases of various mosquito-borne viral infections have increased, and CHIKV infections have been detected in $5 \%$ of patients with dengue-like symptoms, suggesting that surveillance and prevention programs for CHIKV are required (Cha et al., 2013; Hwang and Lee, 2015; Yeom, 2017). In this study, we identified the whole sequence of the structural polyprotein of a CHIKV isolated in Korea, $\mathrm{KNIH} / 2009 / 77$, using reverse transcription. In addition, similar to a previous report analyzing the E1 sequence, we submitted our structural polyprotein sequence to an NCBIBLAST search and phylogenetic analysis and confirmed that the KNIH/2009/77 isolate is clustered within ECSA genotype of CHIKV (Cha et al., 2013).

To evaluate the titer of neutralizing antibodies in a tested serum, the PRNT has been conventionally used in laboratories. Although it is considered a gold standard for neutralization assays, the use of hazardous live CHIKV and the requirement of a BSL-3 facility have impeded research for treatment of diseases caused by this virus. In addition, it takes a relatively long time (about 4-5 days) to visualize and count the plaques for the PRNT. To overcome these limitations, alternative neutralization assays using non-infectious virus replicon particles (VRPs) or pseudotyped viruses have been developed (Gläsker et al., 2013; Kishishita et al., 2013; Hu et al., 2014; Lee et al., 2014). Here, we adopted the strategy of expressing the entire structural polyprotein of CHIKV to generate the pseudotyped virus form of KNIH/2009/77 (CHIKVpseudo) for the enhanced infectivity compared to a virion expressing either an individual E1 or E2 protein (Hu et al., 2014). In addition to increased safety, the PBNA using CHIKVpseudo takes only 2 days to evaluate the neutralizing activity of a tested antibody and makes it easy to quantify the infectivity level using the luciferase activity of infected cells. Furthermore, CHIKVpseudo has additional advantages when compared to other pseudotyped virus systems. First, CHIKVpseudo expresses a FLAG-tagged capsid protein that can be easily detected by a commercially available antibody against FLAG, so that one does not need to generate or use a specific antibody against CHIKV proteins to check expression. Second, we used a dual reporter gene construct that expresses both GFP and luciferase in an infected cell. Many studies using pseudotyped viruses or VLPs often titrate viral infectivity using time- and labor-intensive methods, like measuring the viral genome copy number or virion protein concentration, as is the case for the HIV-1 protein p24 (Salvador et al., 2009; Gläsker et al., 2013; Kishishita et al., 2013; Wichit et al., 2017). Compared to these cases, CHIKVpseudo can be more easily titrated by measuring the GFP signal or luciferase activity. Since GFP is expressed only in infected cells, GFP-positive foci can serve as infectious centers similar to the plaques formed by infectious CHIKV. In addition, we validated the PBNA using CHIKVpseudo in terms of precision, linearity, and specifi- city by comparison with the conventional PRNT. The calculated $\mathrm{IC}_{50}$ and validation values were comparable in both assays despite differences in cell types and incubation times. These results indicate that PBNA with CHIKVpseudo can be successfully employed in a rapid neutralization assay with improved safety compared to PRNTs. Furthermore, the PBNA results with clinical samples from $\mathrm{CHIKV}$-infected patients indicate that our CHIKVpseudo system can be applied to evaluate the neutralizing activities of human sera. In agreement of our results, a recent study reported an application of pseudotyped CHIKV to assess CHIKV DNA-vaccine candidates in their ability to raise neutralizing antibodies $(\mathrm{Wu}$ et al., 2017). Likewise, CHIKVpseudo can be used for assessment of vaccine efficacy.

Taken together, we have identified the whole structural polyprotein sequence of a Korean isolated CHIKV and its phylogenetic position. Using this sequence, we constructed CHIKVpseudo containing the entire structural polyprotein and developed a pseudovirus-based neutralization assay for CHIKV with improved safety and speed. Validation reports of our PBNA also support its reliability as a neutralization test compared to conventional PRNTs. Clinical samples can be also used for our PBNA to measure the neutralizing activity against CHIKV. These results indicate a potential use of CHIKVpseudo to detect neutralizing antibodies for vaccine efficacy tests as well as during clinical diagnosis.

\section{Acknowledgments}

This research was supported by a grant (16172MFDS272) from the Ministry of Food and Drug Safety in 2016-2018, the National Research Foundation of Korea (NRF) funded by the Ministry of Education (2018R1A2B6001363), and a grant from Korea Ministry of Health and Welfare (HI15C2971). The CHIKV strain isolated in Korea (KNIH/2009/77) was obtained from the National Culture Collection for Pathogens, Republic of Korea. The Luc2P-pLVX-IRES-ZsGreen1 plasmid for CHIKVpseudo was a kind gift from Dr. NamHyuk Cho at Seoul National University, Republic of Korea. Human serum samples used in this paper were originally collected as part of the Dengue Vaccine Initiative (DVI) study conducted in Ouagadougou, Burkina Faso, by Centre MURAZ and AGIR.

\section{References}

Azami, N.A.M., Moi, M.L., and Takasaki, T. 2016. Neutralization assay for chikungunya virus infection: plaque reduction neutralization test. In Chikungunya Virus. pp. 273-282. Springer.

Bréhin, A.C., Rubrecht, L., Navarro-Sanchez, M.E., Maréchal, V., Frenkiel, M.P., Lapalud, P., Laune, D., and Desprès, P. 2008. Production and characterization of mouse monoclonal antibodies reactive to Chikungunya envelope E2 glycoprotein. Virology 371, 185-195.

Carleton, M., Lee, H., Mulvey, M., and Brown, D.T. 1997. Role of glycoprotein PE2 in formation and maturation of the Sindbis virus spike. J. Virol. 71, 1558-1566.

Cha, G.W., Cho, J.E., Lee, E.J., Ju, Y.R., Han, M.G., Park, C., and Jeong, Y.E. 2013. Travel-associated Chikungunya cases in South 
Korea during 2009-2010. Osong Public Health Res. Perspect. 4, 170-175.

Chang, K.S., Kim, G.-H., Ha, Y.R., Jeong, E.K., Kim, H.C., Klein, T.A., Shin, S.H., Kim, E.J., Jegal, S., Chung, S.J., et al. 2018. Monitoring and Control of Aedes albopictus, a Vector of Zika Virus, Near Residences of Imported Zika Virus Patients during 2016 in South Korea. Am. J. Med. Hyg. 98, 166-172.

Fukumoto, Y., Obata, Y., Ishibashi, K., Tamura, N., Kikuchi, I., Aoyama, K., Hattori, Y., Tsuda, K., Nakayama, Y., and Yamaguchi, N. 2010. Cost-effective gene transfection by DNA compaction at $\mathrm{pH}$ 4.0 using acidified, long shelf-life polyethylenimine. Cytotechnology 62, 73-82.

Gläsker, S., Lulla, A., Lulla, V., Couderc, T., Drexler, J.F., Liljeström, P., Lecuit, M., Drosten, C., Merits, A., and Kümmerer, B.M. 2013. Virus replicon particle based Chikungunya virus neutralization assay using Gaussia luciferase as readout. Virol. J. 10, 235.

Hu, D., Zhang, J., Wang, H., Liu, S., Yu, L., Sun, L., and Qu, Y. 2014. Chikungunya virus glycoproteins pseudotype with lentiviral vectors and reveal a broad spectrum of cellular tropism. PLoS One 9, e110893.

Hwang, J.H. and Lee, C.S. 2015. The first imported case infected with chikungunya virus in Korea. Infect. Chemother. 47, 55-59.

Jain, M., Rai, S., and Chakravarti, A. 2008. Chikungunya: a review. Trop. Doct. 38, 70-72.

Jose, J., Snyder, J.E., and Kuhn, R.J. 2009. A structural and functional perspective of alphavirus replication and assembly. Fut. Microbiol. 4, 837-856.

Kishishita, N., Takeda, N., Anuegoonpipat, A., and Anantapreecha, S. 2013. Development of a pseudotyped lentiviral vector-based neutralization assay for chikungunya virus infection. J. Clin. Microbiol. 51, 1385-1395.

Kuo, S.C., Chen, Y.J., Wang, Y.M., Tsui, P.Y., Kuo, M.D., Wu, T.Y., and Lo, S.J. 2012. Cell-based analysis of Chikungunya virus E1 protein in membrane fusion. J. Biomed. Sci. 19, 44.

Lee, H.J., Min, K.I., Park, K.H., Choi, H.J., Kim, M.K., Ahn, C.Y., Hong, Y.J., and Kim, Y.B. 2014. Comparison of JEV neutralization assay using pseudotyped JEV with the conventional plaquereduction neutralization test. J. Microbiol. 52, 435-440.

Li, L., Jose, J., Xiang, Y., Kuhn, R.J., and Rossmann, M.G. 2010. Structural changes of envelope proteins during alphavirus fusion. Nature 468, 705.

Morrison, T.E. 2014. Re-emergence of chikungunya virus. J. Virol. 88, 11644-11647.

Mulvey, M. and Brown, D.T. 1995. Involvement of the molecular chaperone $\mathrm{BiP}$ in maturation of Sindbis virus envelope glycoproteins. J. Virol. 69, 1621-1627.

Noranate, N., Takeda, N., Chetanachan, P., Sittisaman, P., A-nuegoonpipat, A., and Anantapreecha, S. 2014. Characterization of chikungunya virus-like particles. PLoS One 9, e108169.

Pal, P., Dowd, K.A., Brien, J.D., Edeling, M.A., Gorlatov, S., Johnson, S., Lee, I., Akahata, W., Nabel, G.J., Richter, M.K., et al. 2013. Development of a highly protective combination monoclonal antibody therapy against Chikungunya virus. PLoS Pathog. 9, e1003312.

Powers, A.M. 2018. Vaccine and therapeutic options to control chikungunya virus. Clin. Microbiol. Rev. 31, e00104-16.

Powers, A.M., Brault, A.C., Tesh, R.B., and Weaver, S.C. 2000. Reemergence of Chikungunya and O'nyong-nyong viruses: evidence for distinct geographical lineages and distant evolutionary relationships. J. Gen. Virol. 81, 471-479.

Rezza, G., Nicoletti, L., Angelini, R., Romi, R., Finarelli, A.C., Panning, M., Cordioli, P., Fortuna, C., Boros, S., Magurano, F., et al. 2007. Infection with chikungunya virus in Italy: an outbreak in a temperate region. Lancet 370, 1840-1846.

Salvador, B., Zhou, Y., Michault, A., Muench, M.O., and Simmons, G. 2009. Characterization of Chikungunya pseudotyped viruses: Identification of refractory cell lines and demonstration of cellular tropism differences mediated by mutations in E1 glycoprotein. Virology 393, 33-41.

Sam, I.C., Loong, S.K., Michael, J.C., Chua, C.L., Sulaiman, W.Y.W., Vythilingam, I., Chan, S.Y., Chiam, C.W., Yeong, Y.S., and AbuBakar, S. 2012. Genotypic and phenotypic characterization of Chikungunya virus of different genotypes from Malaysia. PLoS One 7, e50476.

Schwartz, O. and Albert, M.L. 2010. Biology and pathogenesis of chikungunya virus. Nat. Rev. Microbiol. 8, 491.

Snyder, A.J. and Mukhopadhyay, S. 2012. The alphavirus E3 glycoprotein functions in a clade-specific manner. J. Virol. 86, 1360913620.

Solignat, M., Gay, B., Higgs, S., Briant, L., and Devaux, C. 2009. Replication cycle of chikungunya: a re-emerging arbovirus. Virology 393, 183-197.

Tamura, K., Peterson, D., Peterson, N., Stecher, G., Nei, M., and Kumar, S. 2011. MEGA5: molecular evolutionary genetics analysis using maximum likelihood, evolutionary distance, and maximum parsimony methods. Mol. Biol. Evol. 28, 2731-2739.

Tharmarajah, K., Mahalingam, S., and Zaid, A. 2017. Chikungunya: vaccines and therapeutics. F1000Res. 6, 2114

Thiboutot, M.M., Kannan, S., Kawalekar, O.U., Shedlock, D.J., Khan, A.S., Sarangan, G., Srikanth, P., Weiner, D.B., and Muthumani, K. 2010. Chikungunya: a potentially emerging epidemic? PLoS Negl. Trop. Dis. 4, e623.

Volk, S.M., Chen, R., Tsetsarkin, K.A., Adams, A.P., Garcia, T.I., Sall, A.A., Nasar, F., Schuh, A.J., Holmes, E.C., Higgs, S., et al. 2010. Genome-scale phylogenetic analyses of chikungunya virus reveal independent emergences of recent epidemics and various evolutionary rates. J. Virol. 84, 6497-6504.

Voss, J.E., Vaney, M.C., Duquerroy, S., Vonrhein, C., Girard-Blanc, C., Crublet, E., Thompson, A., Bricogne, G., and Rey, F.A. 2010. Glycoprotein organization of Chikungunya virus particles revealed by X-ray crystallography. Nature 468, 709-712.

Weaver, S.C., Osorio, J.E., Livengood, J.A., Chen, R., and Stinchcomb, D.T. 2012. Chikungunya virus and prospects for a vaccine. Expert. Rev. Vaccines 11, 1087-1101.

Wichit, S., Hamel, R., Bernard, E., Talignani, L., Diop, F., Ferraris, P., Liegeois, F., Ekchariyawat, P., Luplertlop, N., and Surasombatpattana, P. 2017. Imipramine inhibits Chikungunya virus replication in human skin fibroblasts through interference with intracellular cholesterol trafficking. Sci. Rep. 7, 3145.

Wu, J., Zhao, C., Liu, Q., Huang, W., and Wang, Y. 2017. Development and application of a bioluminescent imaging mouse model for Chikungunya virus based on pseudovirus system. Vaccine 35, 6387-6394.

Yap, M.L., Klose, T., Urakami, A., Hasan, S.S., Akahata, W., and Rossmann, M.G. 2017. Structural studies of Chikungunya virus maturation. Proc. Natl. Acad. Sci. USA 114, 13703-13707.

Yeom, J.S. 2017. Current status and outlook of mosquito-borne diseases in Korea. J. Korean Med. Assoc. 60, 468-474. 